\title{
Controlador de servomotores industriales mediante un microcontrolador utilizando MicroPython
}

\section{Industrial servomotor controller by means of a microcontroller using MicroPython}

\author{
RODRÍGUEZ-PONCE, Rafael $\dagger^{*}$ \\ Universidad Politécnica de Guanajuato
}

ID $1^{\text {er }}$ Autor: Rafael, Rodríguez-Ponce / ORC ID: 0000-0001-5006-5580, Researcher ID Thomson: G-5028-2018, arXiv Author ID: rrodriguez3168, CVU CONACYT ID: 209261

DOI: 10.35429/JSL.2019.18.6.15.23

Recibido 23 Enero, 2019; Aceptado 30 Marzo, 2019

\begin{abstract}
Resumen
Los motores eléctricos son máquinas indispensables en la industria, por ello es necesario asegurar que estudiantes de ingeniería puedan desarrollar controladores de velocidad o del par, de manera rápida y eficiente. Una de las estrategias de control de motores mayormente utilizada es Control Vectorial, sin embargo su implementación requiere de un intensivo procesamiento matemático y de un procesador con amplios recursos de computación y velocidad. Se han publicado una gran cantidad de trabajos acerca de este tema, no obstante son dificiles de replicar y de utilizar debido a los problemas anteriormente mencionados. En este artículo el autor presenta un controlador de par y velocidad para servomotores industriales utilizando la estrategia de Control Directo del Par, la cual se ha distinguido por su rápidez y facilidad de implementación, en comparación con Control Vectorial. Además, se utiliza el lenguaje de programación MicroPython en el nuevo y novedoso microcontrolador ESP32, lo cual permite una mayor simplicidad en el diseño. Mediante simulaciones y una implementación física, se validaron los resultados obtenidos lograndose un funcionamiento adecuado del motor. Este trabajo brindará a estudiantes e investigadores interesados en el control de motores industriales, una herramienta rápida y efectiva de implementación.
\end{abstract}

Control de motores eléctricos trifásicos industriales, lenguaje de computación Python, procesador ESP32

\begin{abstract}
Electrical motors are indispensable machines in the industry, thus it is necessary to ensure that engineering students can develop speed and torque controllers, in a fast and efficient manner. One of the most commonly used motor control strategies is Field Oriented Control; however it's implementation requieres highly-intensive math processing and a fast processor with robust hardware resources. Several related works have been published, but they are hard to replicate and use due to the aforementioned problems. In this article, the author presents a torque and speed controller for industrial servomotors by means of Direct Torque Control which is known for its speed and ease of implementation compared to Field Oriented Control. In addition, the MicroPython programming language is used on the new and novel ESP32 microcontroller, which allows greater design simplicity. Results were validated using simulations and a physical implementation, thus achieving adequate motor funcionality. This work will provide students and researchers interested in industrial motor control, a fast and effective implementation tool.
\end{abstract}

Industrial threephase electric motor control, Python computer language, ESP32 processor

Citación: RODRÍGUEZ-PONCE, Rafael. Controlador de servomotores industriales mediante un microcontrolador utilizando MicroPython. Revista de Simulación y Laboratorio. 2019, 6-18: 15-23

\footnotetext{
*Correspondencia al Autor (Correo electrónico: rrodriguez@upgto.edu.mx)

$\dagger$ Investigador contribuyendo como primer Autor
} 


\section{Introducción}

Actualmente en la industría, es de uso común el robot manipulador para manejo de materiales o para cualquier actividad riesgosa para el humano. Principalmente en la industria automotriz, esta máquina es utilizada a gran escala en áreas de estampado, maquinado, soldadura, pintura y ensamble, entre otras. Según cifras de la Federación Internacional de Robotica, para el año 2020 existirán alrededor de 3 millones de robots industriales a nivel mundial y esa cifra incrementa $12 \%$ cada año (IFR, 2018). Por esta razón, es importante que los estudiantes graduados de carreras de Robótica o Mecatrónica tengan un buen entendimiento de su funcionamiento y control.

El motor síncrono de imán permanente (PMSM, del inglés Permanent Magnet Synchronous Motor) es un motor especializado que forma parte integral de la mayoría de robots industriales (Gürocak, 2016). Este motor cuenta con fuertes imanes en el rotor, lo cual le brinda características de alto par y potencia con un tamaño más reducido. Además, comparado con otros motores industriales, tal como el motor asíncrono o jaula de ardilla, es más fácil controlar su velocidad y dirección de rotación (Gieras, 2010).

Para controlar los movimientos del motor PMSM, es necesario un controlador electrónico de alto desempeño, el cual puede utilizar diferentes estrategias de control, siendo la más popular el control de campo orientado (FOC, del inglés Field Oriented Control), comunmente denominada Control Vectorial. Este método de control fue desarrollado a fines de los años sesenta, y desde entonces ha formado parte de la mayoría de controladores de motores industriales debido a que permite un amplio rango de control de velocidad, con una baja pérdida de par (Liu, Li y Zheng, 2018).

Sin embargo, su implementación es bastante complicada al requerir de amplios cálculos matemáticos, por lo que es necesario un procesador de alto desempeño para lograr un control eficiente (Shah y Prakash, 2017). Además, también se requiere tener conocimiento de varios parámetros eléctricos del motor para un funcionamiento apropiado (Huang, Wu y Turner, 2002).
Una alternativa más sencilla y efectiva a FOC, es el control directo del par (DTC, del inglés Direct Torque Control). Esta estrategia de control de motores no es tan precisa como FOC, sin embargo puede controlar los movimientos más rapidamente al requerir una menor cantidad de operaciones matemáticas (Liu, Li y Zheng, 2018). De esta manera, no precisa de un procesador tan rápido y robusto. Además, de los parametros eléctricos del motor, solo es dependiente de la resistencia del estator. Debido a esto, se ha convertido en una herramienta muy útil para el control de motores industriales. Esta técnica de control es parte medular de controladores de motores eléctricos de la marca ABB (Jääskeläinen y Pohjalainen, 2015).

Existe diversas investigaciones en el control de motores trifásicos, ya sea utilizando las estrategias FOC o DTC (Casadei et al., 2002). Normalmente estos trabajos utilizan un procesador de señales digitales (DSP, del inglés Digital Signal Processor) o una matriz reconfigurable de compuertas (FPGA, del inglés Field Programable Gate Array). Tanto el DSP como el FPGA son herramientas muy eficientes para el procesamiento de señales de un motor PMSM, sin embargo la programación se realiza normalmente en lenguaje $\mathrm{C}++\mathrm{o}$ en VHDL, respectivamente, lo cual puede ser presentar un reto considerable, principalmente para programadores con poca experciencia en sistemas embebidos.

En este documento se presenta el desarrollo de un controlador electrónico del par para motores PMSM utilizando la estrategia DTC. Para el procesamiento de las señales se utiliza un novedoso microcontrolador de 32 bits, el cual cuenta con una gran cantidad de recursos de procesamiento, aunque menores que un DSP, sin embargo presenta la ventaja de poderse programar mediante el lenguaje MicroPython. Al igual que Python, MicroPython es un lenguaje muy popular actualmente debido a que presenta una sintáxis muy sencilla de entender, aun para programadores inexpertos (Lepuschitz, 2018). De igual manera, cuenta con una gran cantidad de librerías en línea que facilitan la implementación de cualquier proyecto de procesamiento de señales. Esto será de gran interés para estudiantes de ingeniería o investigadores que requieren controlar un PMSM, de manera fácil y rápida sin la necesidad de hardware especializado. 


\section{Estrategias de control de motores}

Como se mencionó anteriormente, existen diversas estrategias para el control de motores industriales. Una de las más sencillas es el control escalar o también llamado control V/Hz (Smith et al., 2013). En este método se cambia la frecuencia del motor así como su voltaje de alimentación, manteniendo siempre la misma razón. De esta manera se cambia la velocidad síncrona del motor, por lo que también permite cambiar la velocidad y el par del mismo. Aunque los controladores que utilizan esta técnica son sencillos y económicos, también son muy ineficientes debido a que, normalmente son controladores de lazo abierto, y pierden el par cuando la frecuencia es mayor o menor a la frecuencia base (Liu, Li y Zheng, 2018). Este control es normalmente utilizado para motores de inducción en aplicaciones donde no se requiere un control muy preciso.

Para aplicaciones donde se requiere un control más exacto de la velocidad o par de un PMSM, es común utilizar un controlador a base de FOC. La base de esta técnica de lazo cerrado consiste en monitorear constantemente las señales de corriente y voltaje de alimentación del motor para determinar la posición de los vectores de flujo magnético y de corriente del rotor. Orientando estos vectores a una posición perpendicular entre si, se logra obtener el máximo par por unidad de corriente. Sin embargo, para determinar el ángulo de cada uno de los vectores, es necesario aplicar la Transformada de Clarke y de Park, las cuales involucran un intensivo cálculo matemático (LeHuy, 1999).

Además, para aplicar la corrección al rotor, se requiere aplicar la inversa de ambas transformadas matemáticas. Por esta razón estos controladores normalmente requieren un procesador potente que pueda llevar a cabo computaciones complejas a una alta velocidad, de lo contrario se limita la frecuencia de conmutación a valores inferiores a $1 \mathrm{KHz}$, con lo que el comportamiento estático del sistema se ve afectado (Trzynadlowski, 1994).

\section{Control Directo del Par}

La estrategia de control de motores DTC fue desarrollada por Isao Takahashi y Toshihiko Noguchi como una alternativa al popular FOC (Takahashi y Noguchi, 1986).
El objetivo de esta nueva técnica no se basa en el desacoplamiento de los vectores de flujo y corriente como es el caso de FOC, sino más bien se enfoca en controlar el par del motor directamente a través del inversor (VSI, del inglés Voltage Source Inverter). Primeramente, se determinan la magnitud y el ángulo del vector del par electromagnético del rotor con el fin de compararlo contra el par deseado y obtener el error. Mediante una tabla, la cual contiene todos los posibles vectores de corrección, se escoge el vector que más reducirá el error y se aplica directamente al VSI. De esta manera se logra controlar el par del motor de una manera más rápida, ya que no se requieren las computaciones de transformadas de Park o de Clarke (Vas, 1998).

Cabe mencionar que el inconveniente del método de control DTC es la introducción de pequeñas ondulaciones en la corriente denominadas rizo, la cual se produce cuando, al aplicar el vector de voltaje óptimo, el par sobrepasa momentaneamente los límites preestablecidos. Sin embargo, este rizo es normalmente despreciable si los límites de control del par y flujo magnético se escogen adecuadamente, y la frecuencia de muestreo se mantiene a valores mayores a $2 \mathrm{KHz}$ (Llor et al., 2004). En la Figura 1 se muestra un diagrama a bloques simplificado de la técnica DTC.

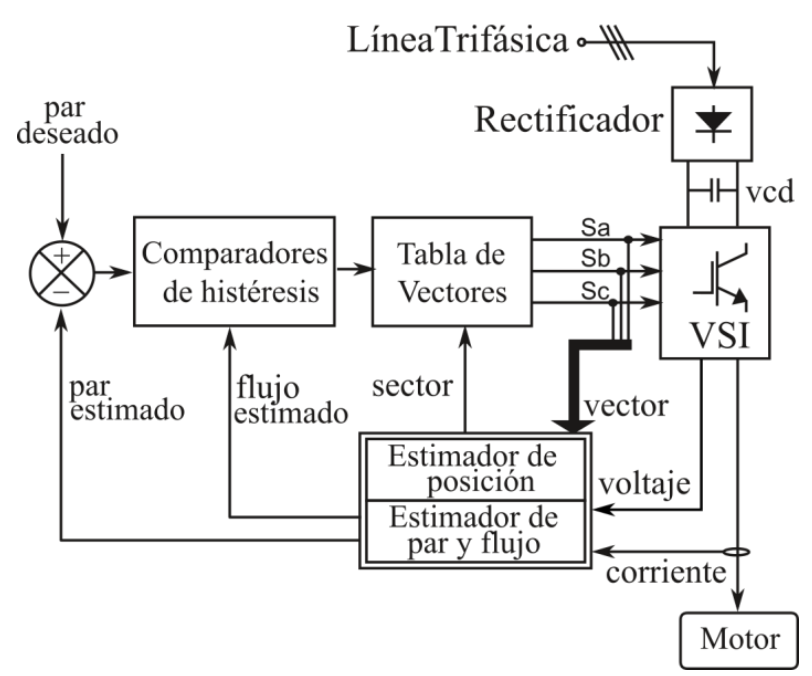

Figura 1 Diagrama a bloques simplificado de DTC Fuente: Elaboración Propia

Se puede observar en el diagrama de DTC, que el proceso inicia tomando muestras de la corriente $\left(I_{a}, I_{b}\right)$ y el voltaje $\left(V_{c d}\right)$ del motor, así como el vector actual del VSI $\left(S_{a}, S_{b}, S_{c}\right)$ y a partir de estos, se determinan las magnitudes del flujo magnético del estator $\left(\varphi_{s}\right)$ y del par electromagnético $\left(T_{e}\right)$. 
Para esto se convierten primeramente de un marco trifásico $(a, b, c)$ a un marco bifásico $(\alpha, \beta)$ como se muestra a continuación:

a) Señales de corriente bifásica:

$I_{\alpha}=I_{a}$

$I_{\beta}=\frac{\sqrt{3}}{3}\left(I_{a}+2 I_{b}\right)$

b) Señales de voltaje bifásico:

$V_{\alpha}=\frac{V_{c d}}{3}\left(2 S_{a}-S_{b}-S_{c}\right)$

$V_{\beta}=\frac{\sqrt{3}}{3} V_{c d}\left(S_{b}-S_{c}\right)$

Enseguida se obtienen los componentes bifásicos del flujo magnético del estator:

$\varphi_{\alpha}=\varphi_{\alpha 0}+\left(V_{\alpha}-R_{s} i_{\alpha}\right) T_{s}$

$\varphi_{\beta}=\varphi_{\beta 0}+\left(V_{\beta}-R_{s} i_{\beta}\right) T_{s}$

Donde, $R_{s}$ es la resistencia del estator, $T_{s}$ es el periodo de muestreo y $\varphi_{\alpha 0}, \varphi_{\beta 0}$ son los componentes de flujo del ciclo previo. A partir de estos, se obtiene la magnitud del flujo magnético del estator $\left(\varphi_{s}\right)$ y el par electromagnético $\left(T_{e}\right)$ como sigue:

$\varphi_{s}=\sqrt{\varphi_{\alpha}^{2}+\varphi_{\beta}^{2}}$

$T_{e}=\frac{3}{2} \frac{P}{2}\left(\varphi_{\alpha} I_{\beta}-\varphi_{\beta} I_{\alpha}\right)$

Donde, $P$ es el número de pares de polos del rotor del motor.

Enseguida, se compara el par obtenido contra la consigna del par, para obtener el error de par $e_{T}$. Además, el valor de la magnitud del flujo se compara contra el valor del flujo del motor y se obtiene el error de flujo $e_{\varphi}$. Ambos errores se analizan para determinar si se encuentran dentro de los límites preestablecidos en dos comparadores de histéresis.

El comparador de histéresis para el flujo (Figura 2) tiene dos posibles valores de salida, $\phi=0$ y $\phi=1$, dependiendo de si el error del flujo rebasó el límite inferior o el superior, respectivamente.

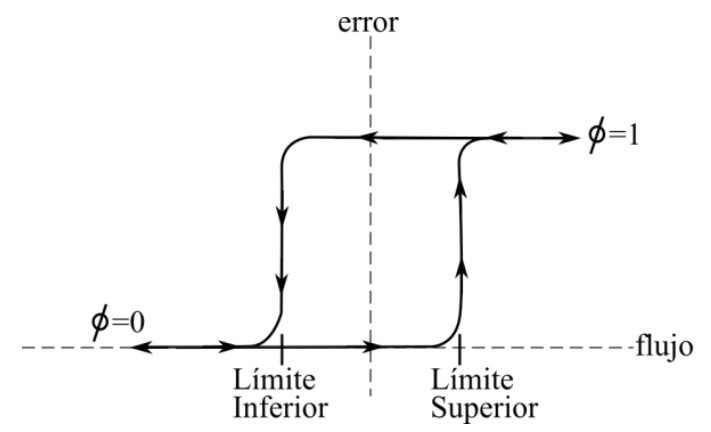

Figura 2 Comparador de histéresis de dos niveles para el error del flujo

Fuente: Elaboración Propia

El comparador de histéresis para el error del par (Figura 3) tiene tres posibles valores de salida, $\tau=1, \tau=-1$ y $\tau=0$, dependiendo de si el error del par rebasó el límite superior, el inferior, $o$ se encuentra entre ambos, respectivamente.

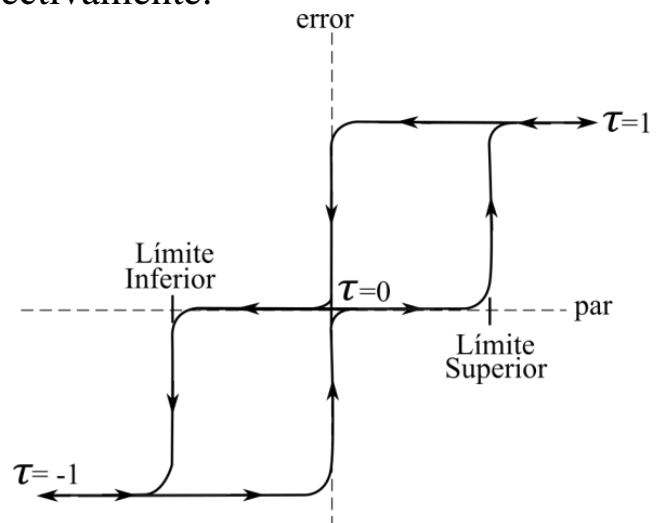

Figura 3 Comparador de histéresis de tres niveles para el error del par

Fuente: Elaboración Propia

Antes de poder determinar el vector óptimo para corregir los errores de par y de flujo, es necesario localizar la posición actual del vector de flujo en el rotor. Para esto, la trayectoria de $360^{\circ}$ del vector se divide en 6 sectores de $60^{\circ}$ cada uno, como se muestra en la Figura 4.

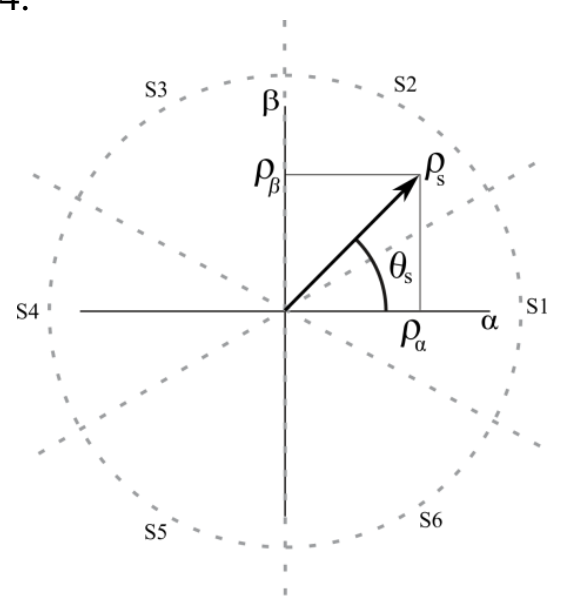

Figura 4 Sectores S1 a S6 para la trayectoria circular del vector de flujo del estator

Fuente: Elaboración Propia 
Para determinar el sector en el cuál se encuentra el vector de flujo, se utilizan los componentes $\varphi_{\alpha}$ y $\varphi_{\beta}$ determinados en (5) y (6), respectivamente, para calcular el ángulo $\theta_{s}$ mediante la siguiente expresión:

$\theta_{s}=\tan ^{-1} \frac{\varphi_{\beta}}{\varphi_{\alpha}}$

Utilizando los valores de salida $(\phi y \tau)$ de ambos comparadores de histéresis y el sector actual del vector de flujo, se determina el vector óptimo que debe ser aplicado al inversor de transistores, tal como se muestra en la Tabla 1.

\begin{tabular}{|c|c|c|c|c|c|c|c|}
\hline \multirow{2}{*}{$\phi$} & \multirow{2}{*}{$\tau$} & \multicolumn{6}{|c|}{ Sector } \\
\hline & & 1 & 2 & 3 & 4 & 5 & 6 \\
\hline \multirow{3}{*}{$\phi=1$} & $\tau=1$ & $\mathrm{~V}_{2}$ & $\mathrm{~V}_{3}$ & $\mathrm{~V}_{4}$ & $\mathrm{~V}_{5}$ & $\mathrm{~V}_{6}$ & $\mathrm{~V}_{1}$ \\
\hline & $\tau=0$ & $\mathrm{~V}_{7}$ & $\mathrm{v}_{0}$ & $V_{7}$ & $\mathrm{v}_{0}$ & $\mathrm{~V}_{7}$ & $\mathrm{v}_{0}$ \\
\hline & $\tau=-1$ & $\mathrm{~V}_{6}$ & $\mathrm{v}_{1}$ & $\mathrm{~V}_{2}$ & $\mathrm{v}_{3}$ & $\mathrm{v}_{4}$ & $\mathrm{~V}_{5}$ \\
\hline \multirow{3}{*}{$\phi=0$} & $\tau=1$ & $\mathrm{~V}_{3}$ & $\mathrm{~V}_{4}$ & $\mathrm{~V}_{5}$ & $\mathrm{~V}_{6}$ & $\mathrm{~V}_{1}$ & $\mathrm{v}_{2}$ \\
\hline & $\tau=0$ & $\mathrm{v}_{0}$ & $\mathrm{v}_{7}$ & $\mathrm{v}_{0}$ & $\mathrm{~V}_{7}$ & $\mathrm{v}_{0}$ & $\mathrm{v}_{7}$ \\
\hline & $\tau=-1$ & $\mathrm{~V}_{5}$ & $v_{6}$ & $\mathrm{~V}_{1}$ & $\mathrm{~V}_{2}$ & $v_{3}$ & $\mathrm{~V}_{4}$ \\
\hline
\end{tabular}

Tabla 1 Vectores óptimos para el inversor Fuente: Elaboración Propia

Cada uno de los vectores de la tabla indica cuál de los transistores de potencia del VSI debe ser encendido o apagado. De esta manera se tienen un total de seis posibles vectores de corrección ( $\left.\mathrm{v}_{1} \mathrm{a} \mathrm{v}_{6}\right)$, ya que con los vectores $\mathrm{v}_{0}$ (000) y v7 (111) no hay paso de corriente hacia el motor, por lo tanto el vector de flujo no cambia con ellos. Por esta razón, estos vectores son denominados los vectores nulos. En la Figura 5 se muestran los posibles vectores de corrección que se aplican al VSI.

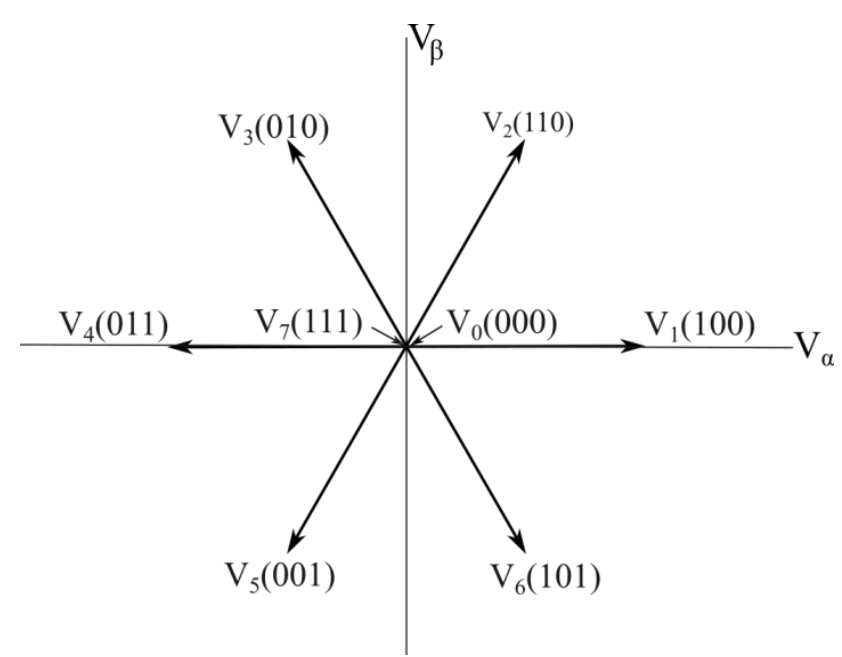

Figura 5 Posición de los posibles vectores de corrección óptimos para el sistema DTC que se aplican al VSI Fuente: Elaboración Propia
Como se mencionó anteriormente, cuando el vector óptimo se aplica al VSI, se genera una corrección inmediata al vector del par, el cual debe mantenerse dentro de los límites establecidos en el comparador de histéresis de tres niveles. Sin embargo, cuando los límites son rebasados, se genera rizo en las señales de par y corriente hasta que un nuevo vector de corrección es generado.

Por esta razón, es importante que la banda de hystéresis no sea demasiado estrecha con el fin de mantener la frecuencia de conmutación arriba de $2 \mathrm{KHz}$ y el rizo dentro de un nivel aceptable (Beerten, Verveckken y Driesen, 2009).

\section{Hardware del servocontrolador DTC}

El procesador que se encarga de la ejecución de DTC es el microcontrolador ESP32 del fabricante ESPRESSIF localizada en Shanghai, China (Espressif, 2019).

Este dispositivo de 32 bits, introducido en el 2016, ha tenido gran aceptación en proyectos de electrónica, control y procesamiento de imágenes debido a sus características de alta velocidad y extensos recursos computacionales, además de un bajo costo comparado con dispositivos similares (Ivkovic e Ivkovic, 2019). En la Tabla 2 se presenta un listado de las características y periféricos principales del procesador.

\begin{tabular}{|l|l|}
\hline \multicolumn{1}{|c|}{ Componentes } & \multicolumn{1}{|c|}{ Descripción } \\
\hline Procesador & $\begin{array}{l}\text { CPU de doble núcleo, 32 bits, 240 } \\
\text { MHz. }\end{array}$ \\
\hline Memoria & 4 MB SPI Flash, 8MB PSRAM. \\
\hline \multirow{3}{*}{ Periféricos } & $\begin{array}{l}\text { 2 x ADC SAR de 12 bits, 2 x DAC de } \\
\text { Timers de 64 bits, 3 x WDT, 34 } \\
\text { terminales GPIO. }\end{array}$ \\
\hline Comunicaciones & $\begin{array}{l}\text { Wi-Fi (2.4 GHz), Bluetooth 4.2, CAN } \\
\text { bus 2.0, 4 x SPI, 3 x UART, 1 DMA, } \\
2 \times I^{2} \mathrm{C} \text { (Inter-IC bus) y 2 x I²S (Inter- } \\
\text { Ic Sound bus). }\end{array}$ \\
\hline
\end{tabular}

Tabla 2 Características y periféricos principales del microcontrolador ESP32

Fuente: Elaboración Propia

Los periféricos mayormente utilizados en este proyecto fueron los convertidores analógico/digital, los de digital/analógico y un timer; los primeros convierten las señales analógicas de corriente $I_{a}$ e $I_{b}$, a valores digitales para iniciar el proceso DTC. 
Los segundos son utilizados para poder observar distintas señales digitales en un osciloscopio, y por último, el timer es utilizado para mantener un tiempo de muestreo constante de $250 \mu \mathrm{s}$. La etapa de potencia del servocontrolador se diseñó con una arquitectura modular en mente, es decir, cada uno de los elementos tiene su propia tarjeta de circuito impreso.

Teniendo en cuenta que el controlador tuviera fines didácticos, los módulos puedan ser intercambiados fácilmente. De esta manera, por ejemplo, el módulo de sensado de corriente mediante sensores inductivos, puede ser intercambiado por uno de sensores de efecto de Hall o diferente, con el fin de observar diferencias de funcionamiento. En cuanto al módulo de procesamiento del algoritmo DTC, se tiene previsto que se puedan utilizar diferentes tecnologías como son tarjetas de desarrollo basadas en DSP o en FPGA.

A continuación, en la Tabla 3 se listan los dispositivos principales, y en la Figura 6 se muestran los módulos del servocontrolador.

\begin{tabular}{|l|lr|}
\hline Componentes & \multicolumn{3}{|c|}{ Descripción } \\
\hline $\begin{array}{l}\text { A501-2001- } \\
\text { 0232 }\end{array}$ & $\begin{array}{l}\text { Rectificador trifásico } \\
\text { ELECTRIC 600V, 20A }\end{array}$ \\
\hline $\begin{array}{l}\text { 6MBI15GS- } \\
\text { 060 }\end{array}$ & $\begin{array}{l}\text { Módulo de 6x IGBTs } \\
\text { ELECTRIC, 600V, 15A }\end{array}$ \\
\hline HCGF4A & $\begin{array}{l}\text { Capacitor electrolítico HITACHI, } \\
1000 \mu F, 400 V d c, \text { surge 450Vdc }\end{array}$ \\
\hline 56300C & $\begin{array}{l}\text { Sensor inductivo MURATA de 300 } \\
\text { vueltas, 10A, 180-335mH @ 1V } \\
10 K H z\end{array}$ \\
\hline IR2110 & $\begin{array}{l}\text { Controlador de disparo IGBTs INT. } \\
\text { RECTIFIER, 500V, 2A }\end{array}$ \\
\hline TL084 & $\begin{array}{l}\text { Amplificador operacional (4X) } \\
\text { TEXAS INSTRUMENTS de entrada } \\
\text { FET }\end{array}$ \\
\hline ESP32 & $\begin{array}{l}\text { Microcontrolador de 32 bits } \\
\text { ESPRESSIF }\end{array}$ \\
\hline
\end{tabular}

Tabla 3 Lista de componentes principales utilizados en el controlador de motores PMSM

Fuente: Elaboración Propia

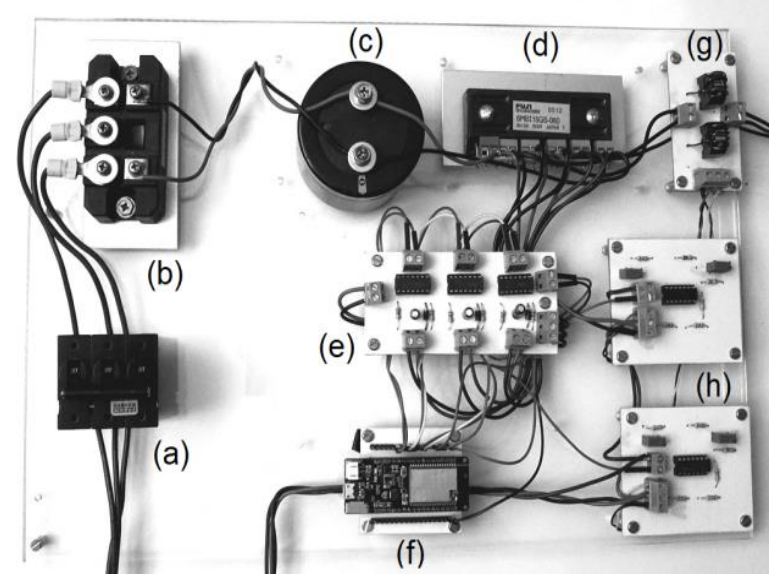

Figura 6 Implementación física del controlador de motores PMSM donde se pueden observar (a) el interruptor principal, (b) el rectificador trifásico, (c) el capacitor de filtrado, (d) puente de IGBTs trifásico, (e) el controlador de disparo de los IGBTs, (f) el microcontrolador ESP32, (g) los sensores inductivos de corriente y (h) los acondicionadores de señal

Fuente: Elaboración Propia

Para las pruebas se utilizó principalmente un motor PMSM industrial de la marca FANUC, modelo 3-OF, cuyos parámetros más relevantes se presentan en la Tabla 4.

\begin{tabular}{|l|r|}
\multicolumn{1}{|c|}{ Parámetros eléctricos } & \multicolumn{1}{c|}{ Valor } \\
\hline Número de pares de polos, $P$ & 6 \\
\hline Resistencia del Estator, $R s$ & $8.4 \Omega$ \\
\hline Flujo magnético, $\varphi_{f}$ & $0.347 \mathrm{~Wb}$ \\
\hline Voltaje máximo, $V$ & $110 \mathrm{~V}$ \\
\hline Corriente máxima, $I$ & $2 \mathrm{~A}$ \\
\hline Velocidad base, $\omega_{b}$ & $5000 \mathrm{rpm}$ \\
\hline
\end{tabular}

Tabla 4 Lista de los parámetros eléctricos más relevantes del motor FANUC

Fuente: Elaboración Propia

\section{Implementación de DTC}

La estrategia de control DTC fue implementada en una subrutina de interrupción (Figura 7) ejecutada cada $250 \mu$ s. Inicia con la conversión de las señales analógicas $\left(I_{a}, I_{b}\right)$ a digital, seguido de un ajuste de su magnitud y offset. Enseguida, se obtienen los valores bifásicos de voltaje, corriente y de flujo. Conviene aclarar que, con el fin de reducir el tiempo de procesamiento a un mínimo, no se aplicó la raiz cuadrada para la estimación del flujo magnético. En su lugar, se elevó al cuadrado la consigna de flujo. Además, en lugar de la conversión de $V_{c d}$ a digital, se utilizó un valor constante a $100 \mathrm{~V}$ y este dato se introdujo directamente en el cálculo de los componentes bifásicos de voltaje. 
\#Subrutina de Interrupción del Timer def isr_DTC(pin):

\# conversión A/D de señales de corriente

$\mathrm{Ia}=\operatorname{adc} 1 \cdot \operatorname{read}()$

$\mathrm{Ib}=\operatorname{adc} 2 \cdot \operatorname{read}()$

\# ajuste de valores de magnitud y offset

$\mathrm{Ia}=(\mathrm{Ia} * 0.001957)-0.5$

$\mathrm{Ib}=(\mathrm{Ib} * 0.001957)-0.5$

\# componentes bifásicos corr. y volt.

Ialfa $=$ Ia

Ibeta $=0.577 *(\mathrm{Ia}+2 * \mathrm{Ib})$

Valfa $=33.3 *(2 * \mathrm{Sa}-\mathrm{Sb}-\mathrm{Sc})$

Vbeta $=57.7 *(\mathrm{Sb}-\mathrm{Sc})$

\#componentes bifásicos flujo magnetico palfa $=$ palfa_0 $+(($ Valfa - Rs*Ialfa $) * T s)$ pbeta $=$ pbeta $0+\left((\text { Vbeta }- \text { Rs*Ibeta })^{*} \mathrm{Ts}\right)$

palfa $0=$ palfa

pbeta_ $0=$ pbeta

\#estimación de flujo magnético y par

flux $=($ palfa $* * 2)+($ pbeta $* * 2)$

$\mathrm{Te}=0.75^{*} \mathrm{P} *($ palfa*Ibeta - pbeta*Ialfa $)$

\#obtención de errores

error_flux $=($ flux_ref**2) - flux

error_par $=$ Te_ref - Te

\#bandas de histéresis

phi $=$ hist flux(error flux)

tau $=$ hist_flux(error_par)

\#cálculo del sector del vector de flujo sector $=$ calc_sector(palfa,pbeta)

\#obtención del vector óptimo

vect $=$ vector_optimo(phi,tau,sector)

\#aplicación del vector a VSI

$\mathrm{Sa}=\operatorname{vect}[0]$

$\mathrm{Sb}=\operatorname{vect}[1]$

$\mathrm{Sc}=\operatorname{vect}[2]$

sal_VSI(Sa,Sb,Sc)

Figura 7 Subrutina de interrupción en lenguaje Micropython programada en el microcontrolador ESP32 para ejecución de DTC

Fuente: Elaboración Propia

Los valores del par y el flujo son comparados contra las consignas respectivas y obtenidos los errores, se aplican, junto con el sector del vector de flujo, a la tabla de vectores. El vector resultante se aplica al VSI.

En el listado de código Micropython mostrado anteriormente, se incluyen solamente las operaciones más relevantes con el fin de limitar la extensión del documento.

\section{Resultados de Simulación de DTC}

La simulación del sistema completo se realizó en MATLAB-Simulink (Figura 8) con el fin de analizar el funcionamiento del controlador bajo diferentes condiciones de tiempo de muestreo y anchuras de las bandas de histéresis, previo a la implementación.

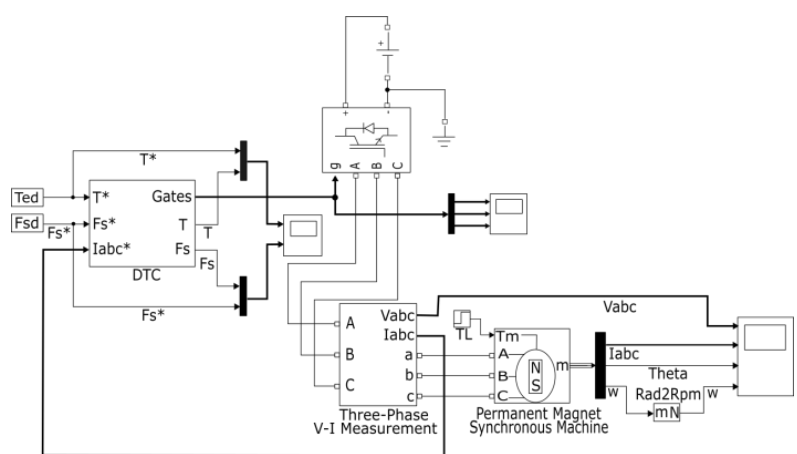

Figura 8 Simulación del sistema DTC en MATLABSimulink

Fuente: Elaboración Propia

Aunque ya existe un módulo DTC completo en Simulink, se diseñó uno completamente nuevo y personalizado, con el fin de que la simulación se ajustará lo más posible a la implementación física. Además esto permitió poder observar el comportamiento dinámico de cada una de las operaciones internas de DTC, lo cual no es posible con el módulo que provee el fabricante. De las señales del proceso, las más importantes a observar, son el flujo magnético y la corriente de salida; con los componentes bifásicos del flujo es posible determinar si los límites de histéresis son aceptables, y en la señal de corriente es posible observar los efectos del rizo.

En la Figura 9 se muestra el lugar geométrico del flujo estatórico, el cual es deseable sea lo más cercano a un círculo, y con ello, la corriente del estator sea lo más cercano a una señal sinusoidal. Si la magnitud de la banda de histeresis de flujo aumenta, la frecuencia de conmutación disminuye y el lugar geométrico del flujo se acerca más a un hexágono, lo cual aumenta la distorción harmónica en la corriente estatórica, causando vibración y ruido en el motor.

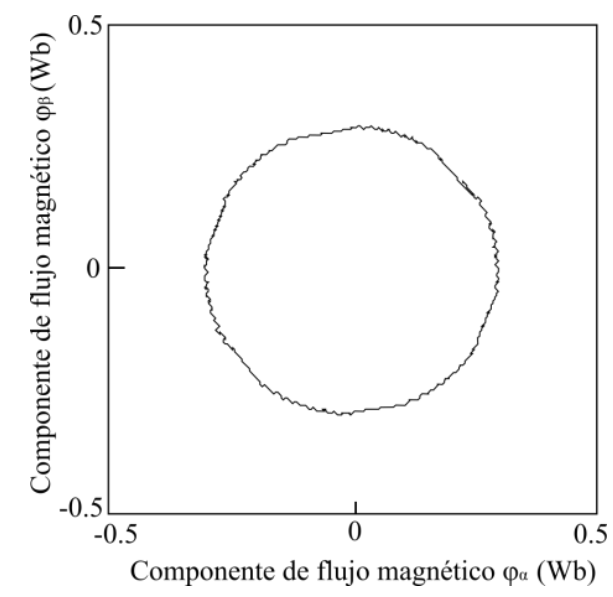

Figura 9 Simulación del lugar geométrico del flujo magnético del estator

Fuente: Elaboración Propia

RODRÍGUEZ-PONCE, Rafael. Controlador de servomotores industriales mediante un microcontrolador utilizando MicroPython. Revista de Simulación y Laboratorio. 2019. 
Se hicieron también múltiples simulaciones para estudiar principalmente, los efectos de la banda de histéresis del flujo. El ancho más adecuado determinado fue un $2 \%$ de la señal de referencia del flujo. En cambio, para el par se utilizó un 5\% de la señal de referencia correspondiente. En la Figura 10 se puede observar una de las fases de corriente $\left(I_{a}\right)$ del motor y se puede apreciar que el valor del rizo es relativamente bajo.

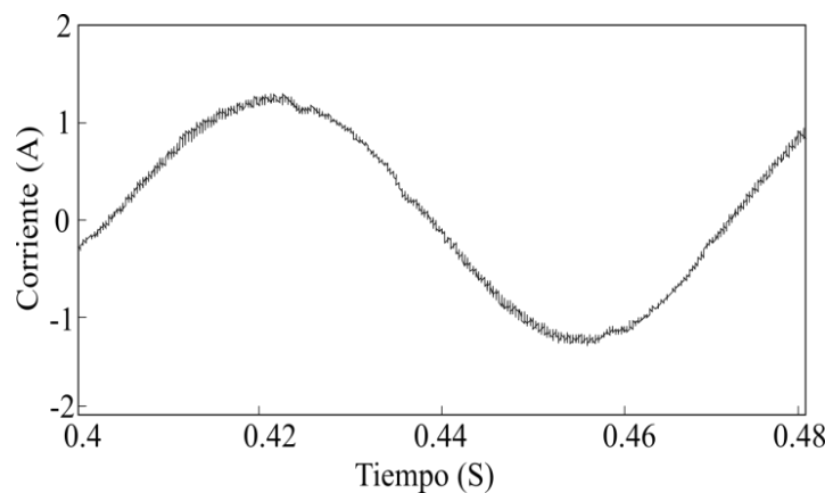

Figura 10 Simulación de la corriente de salida en una fase del motor $\left(I_{a}\right)$

Fuente: Elaboración Propia

\section{Resultados de la implementación práctica}

A continuación, se presentan los resultados experimentales principales obtenidos del comportamiento real del controlador de PMSM.

Primeramente, se tuvieron que convertir las señales digitales de los componentes bifásicos del flujo a señales analógicas para poder observar el lugar geométrico estatorico. Este se muestra en la Figura 11, en donde se puede observar que se aproxima a un círculo y el contenido de rizo es relativamente bajo.

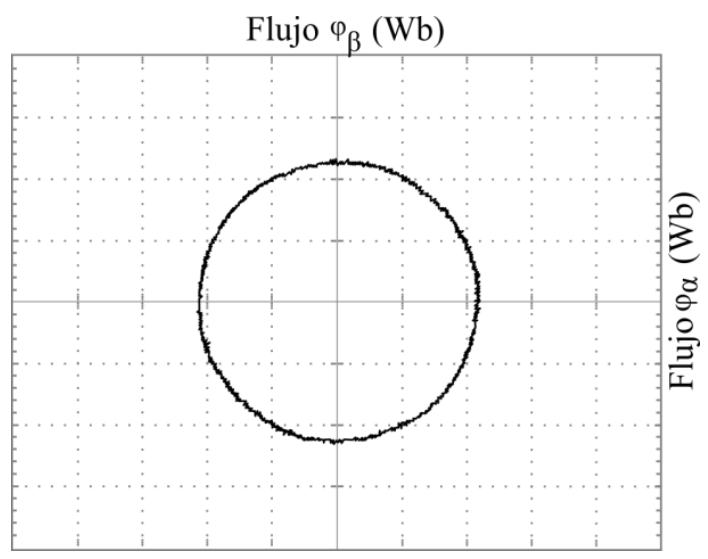

Figura 11 Lugar geométrico del flujo magnético del estator $(0.1 \mathrm{~Wb} / \mathrm{div})$

Fuente: Elaboración Propia
La señal de la intensidad de corriente de una de las fases del motor (Figura 12) muestra una calidad bastante alta ya que tiene una forma claramente sinusoidal con un bajo contenido de rizo. Esto se tradujo en una operación silenciosa y sin vibración en el motor PMSM, a diferentes velocidades de operación y bajo distintas cargas.

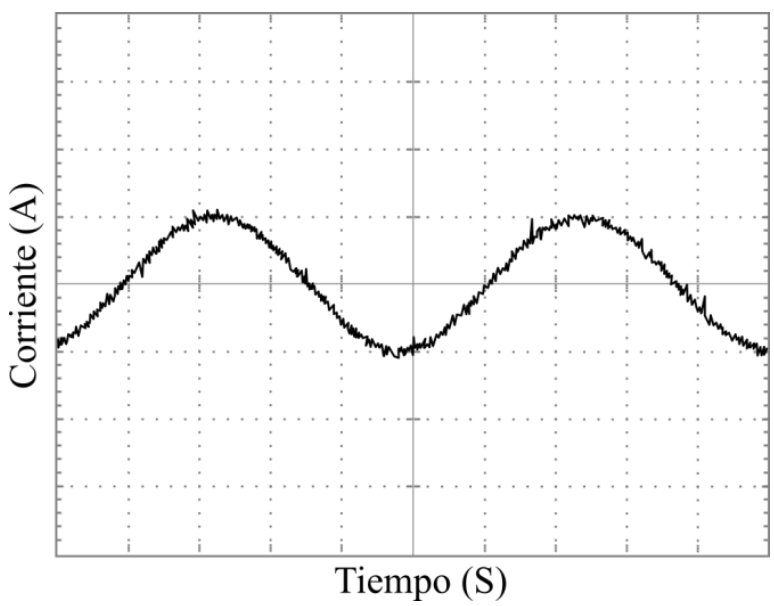

Figura 12 Corriente del estator Ia (1 A/div, 5ms/div) Fuente: Elaboración Propia

Por último, en la Figura 13 se muestra la respuesta del par electromagnético del controlador, en donde se muestran la consigna del par $( \pm 0.25 \mathrm{Nm})$ y el par de salida.

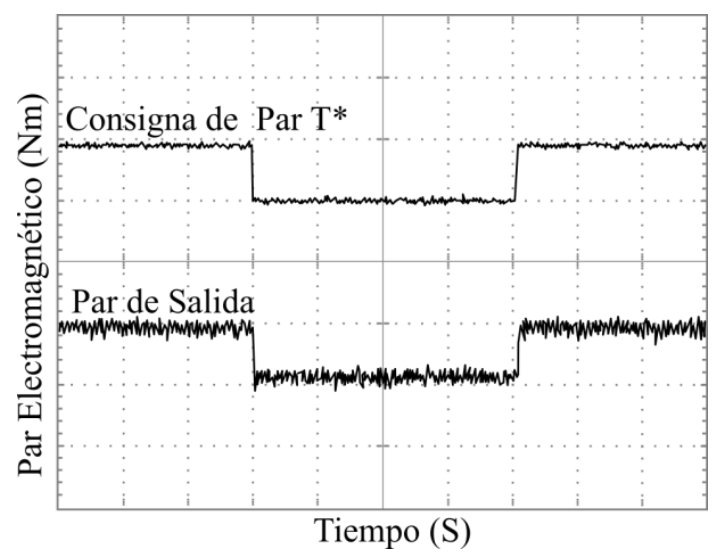

Figura 13 Respuesta del par electromagnético (0.5 $\mathrm{Nm} / \mathrm{div}, 0.3 \mathrm{~s} / \mathrm{div}$ )

Fuente: Elaboración Propia

\section{Conclusión}

En este trabajo se presentó un controlador de motores industriales PMSM con base en el microcontrolador ESP32 y el lenguaje de programación Micropython. Mediante la aplicación de la estrategia DTC, se obtuvo un controlador con un eficiente desempeño dinámico y un bajo contenido de rizo, gracias a un bajo tiempo de muestreo de $250 \mu \mathrm{s}$. 
Los resultados obtenidos fueron validados mediante simulación electrónica en MatlabSimulink, así como en una implementación física en un servocontrolador modular escalable.

Este trabajo será de gran interés para estudiantes o investigadores en busca de una alternativa sencilla y eficiente, a la estrategia de control vectorial.

\section{Referencias}

Beerten, J., Verveckken, J. y Driesen, J. (2009, mayo). Comparison of Three-Level Torque Hysteresis Controllers for Direct Torque Control. IEEE Eurocon, St.-Petersburg, Rusia.

Casadei, D., Profumo, F., Serra. G. y Tani., A. (2002). FOC and DTC: two viable schemes for induction motors torque control. IEEE Trans. Pwr. Electr., 17(5), 779-787.

Espressif. (2019). ESP32 Overview. Shanghai, China, recuperado de https://www.espressif. com/en/products/hardware/esp32/overview.

Gieras, J. (2010). Permanent Magnet Motor Technology: Design and Applications. Boca Raton: Taylor and Francis Group.

Gürocak, H. (2016). Industrial Motion Control: Motor Selection, Drives, Control Tuning, Applications. West Sussex, UK: John Wiley \& Sons Ltd.

Huang, K.S., Wu, Q.H. y Turner, D.R. (2002). Effective identification of induction motor parameters based on fewer measurements. IEEE Trans. Energy Conv., 17(1), 55-60.

International Federation of Robotics (IFR). (2018, marzo). Robots and the workplace of the future. International Federation of Robotics, 135 , recuperado de https://ifr.org/papers.

Ivkovic, J. e Ivkovic, J.L. (2017). Analysis of the Performance of the New Generation of 32-bit Microcontrollers for IoT and Big Data Application. $7^{\text {th }}$ International Conference on Information Society and Technology ICIST, Kapaonik, Serbia.

Jääskeläinen, E. y Pohjalainen, P. (2015). DTC, A motor control technique for all seasons. $A B B$ White papers, 1-12, recuperado de https://library.abb.com/en/results.
Le-Huy, H. (1999, octubre). Comparison of field-oriented control and direct torque control for induction motor drives. IEEE Industry Applications Conference. Phoenix, USA.

Llor, A., Allard, B., Lin-Shi, X. y Retif, J.M. (2004). Comparison of DTC implementations for synchronous machines. IEEE Power Electronics Specialist Conference. Aachen, Alemania.

Lepuschitz, W. (2018). Robotics in Education. Latest Results and Developments. Springer International Publishing.

Liu, Z., Li, Y. y Zheng, Z. (2018). A Review of Drive Techniques for Multiphase Machines. IEEE Trans. Electr. Mach. Syst., 2(2), 243-251.

Maier, A., Sharp, A. y Vagapov, Y. (2017). Comparative analysis and practical implementation of the ESP32 microcontroller module for the internet of things. Internet Technologies and Applications ITA. Wrexham, UK.

Shah, V. y Prakash, S. (2017, noviembre). FPGA Implementation of Sensorless Field Oriented Current Control of Induction Machine. IEEE International Conference on Computational Intelligence and Computing Research. Coimbatore, India.

Smith, A., Gadoue, S., Armstrong, M. y Finch, J. (2013). Improved method for the scalar control of induction motor drives. IET Power Electronics Applications, 7(6), 487-498.

Takahashi, I. y Noguchi, T. (1986). A New Quick-Response and High-Efficiency Control Strategy of an Induction Motor. IEEE Trans. Ind. Appl., 22(5), 820-827.

Trzynadlowski, A. (1994). The Field Oriented Principle in Control of Induction Motors. New York: Springer.

Vas, P. (1998). Sensorless Vector and Direct Torque Control. New York: Oxford University Press. 